\title{
SERS active colloidal nanoparticles for the detection of small blood biomarkers using aptamers
}

\author{
Haley Marks ${ }^{1}$, Samuel Mabbott ${ }^{4}$, George W. Jackson ${ }^{2,3}$, Duncan Graham ${ }^{4}$, Gerard L. Coté ${ }^{1}$ \\ ${ }^{1}$ Department of Biomedical Engineering, Texas A\&M University, College Station, TX \\ ${ }^{2}$ BioTex, Inc., Houston, Texas, United States \\ ${ }^{3}$ Base Pair Biotechnologies, Inc., Pearland, Texas, United States \\ ${ }^{4}$ Department of Pure and Applied Chemistry, University of Strathclyde, Glasgow, Scotland
}

\begin{abstract}
Functionalized colloidal nanoparticles for SERS serve as a promising multifunctional assay component for blood biomarker detection. Proper design of these nanoprobes through conjugation to spectral tags, protective polymers, and sensing ligands can provide experimental control over the sensitivity, range, reproducibility, particle stability, and integration with biorecognition assays. Additionally, the optical properties and degree of electromagnetic SERS signal enhancement can be altered and monitored through tuning the nanoparticle shape, size, material and the colloid's local surface plasmon resonance (LSPR). Aptamers, synthetic affinity ligands derived from nucleic acids, provide a number of advantages for biorecognition of small molecules and toxins with low immunogenicity. DNA aptamers are simpler and more economical to produce at large scale, are capable of greater specificity and affinity than antibodies, are easily tailored to specific functional groups, can be used to tune inter-particle distance and shift the LSPR, and their intrinsic negative charge can be utilized for additional particle stability ${ }^{1,2}$. Herein, a "turn-off" competitive binding assay platform involving two different plasmonic nanoparticles for the detection of the toxin bisphenol A (BPA) using SERS is presented. A derivative of the toxin is immobilized onto a silver coated magnetic nanoparticle (Ag@MNP), and a second solid silver nanoparticle (AgNP) is functionalized with the BPA aptamer and a Raman reporter molecule (RRM). The capture $(\mathrm{Ag} @ \mathrm{MNP})$ and probe (AgNP) particles are mixed and the aptamer binding interaction draws the nanoparticles closer together, forming an assembly that results in an increased SERS signal intensity. This aptamer mediated assembly of the two nanoparticles results in a 100x enhancement of the SERS signal intensity from the RRM. These pre-bound aptamer/nanoparticle conjugates were then exposed to picomolar levels of BPA in free solution and the competitive binding event was monitored by the decrease in SERS intensity.
\end{abstract}

Keywords: Surface enhanced Raman spectroscopy (SERS), aptamer, plasmonic nanoparticles, competitive binding assay, molecular diagnostics

\section{INTRODUCTION}

Molecularly mediated surface enhanced Raman spectroscopy (SERS) can be a desirable approach for ultrasensitive blood biomarker detection due to its high sensitivity and capability for multiplexing. SERS analysis of DNA-directed nanoparticle assembly using functionalized colloidal nanoparticles has been used extensively as a method to measure and control the degree of enhancement of Raman scattering from a Raman active dye in response to a molecular binding event ${ }^{3}$. SERS assays in both "turn -on" and "turn-off" formats have been developed using oligonucleotides to detect DNA fragments ${ }^{4}$, as well as with aptamers for the detection of biomarker proteins ${ }^{5}$. In this work we present a "SERS off" competitive binding assay for the detection of BPA: a small molecule toxic to the human endocrine system commonly found in food and beverage packaging.

A schematic representation of the two nanoprobes developed for this assay is shown in Figure 1. The 'target' silver nanoparticle is functionalized with a mixed self-assembled monolayer consisting of the Raman reporter molecule malachite green isothiocyanate (MGICT) and BPA aptamers. These particles are designed to bind to a version of the toxin bisphenol A diglycidyl ether (BADGE), immobilized onto a second silver coated magnetic 'probe' nanoparticle, where binding affinities have been determined previously by Marks et $\mathrm{al}^{6}$. The assembled nanoparticle network provides a localized 'hot spot' of electric field enhancement around the RRM, and therefore an increase in the number of scattering 
events experienced by the Raman reporter that is observable in the SERS spectral intensity. Upon introduction of free BPA in its native form, the bound AgNPs are competitively freed; reversing the nanoparticle assembly and causing the SERS signal to "turn-off" and decrease in response to the competitive binding event.

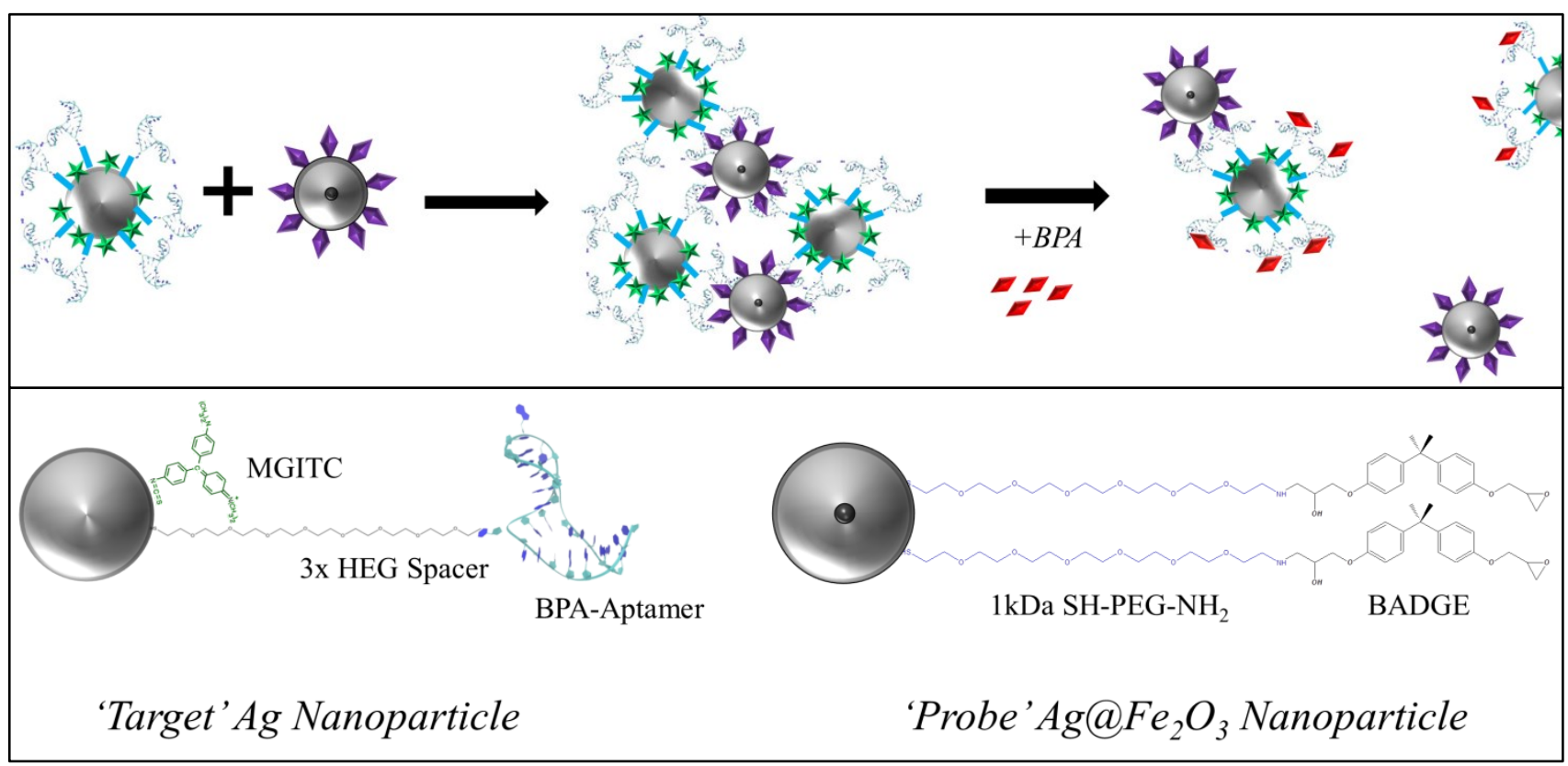

Figure 1. Top: Schematic illustration of the aptamer mediated 'turn-off' SERS competitive binding assay. Bottom: Target and probe nanoparticle conjugate components.

\section{MATERIALS \& METHODS}

\subsection{Materials and Instrumentation}

The modified BPA aptamer': 5' - [ThiSS][HEG] 3 CCG CCG TTG GTG TGG TGG GCC TAG GGC CGG CGG CGC ACA GCT GTT ATA GAC GTC TCC AGC-3' was synthesized by Eurofins Genomics (Germany). Hetero-bifunctional PEG linker (NH2-PEG-SH, 1kDa) was purchased from NanoCS (USA). Malachite green isothiocyanate reporter dye was purchased from Invitrogen (UK). All other reagents were obtained from Sigma Aldrich (USA/UK).

A Varian Cary 300Bio UV-Visible Spectrophotometer was used with a scan range of 200-800 nm for extinction measurements. The zeta potential and hydrodynamic diameters of the nanoparticles were measured on a Zetasizer Nano ZS90 (Malvern, U.K.). SERS measurements were collected using a WiTec Raman instrument equipped with a 532 nm laser.

\subsection{Colloid Synthesis}

Silver colloid (AgNP) was synthesized using the reduction method as reported by Leopold and Lendl ${ }^{8}$. Hydroxylamine hydrochloride $(1 \mathrm{ml}, 150 \mathrm{mM})$ was added to $89 \mathrm{~mL}$ of $\mathrm{NaOH}(3.33 \mathrm{mM})$ under vigorous stirring. Silver nitrate $\left(\mathrm{AgNO}_{3}\right)$ solution $(10 \mathrm{mM})$ was added drop-wise and stirred for $15 \mathrm{~min}$ at room temperature. DLS measurements revealed an average particle diameter of $\sim 45 \mathrm{~nm}$ and the stock particle concentration was determined to be $145 \mathrm{pM}$ according to Beer's Law using an extinction coefficient of $2.87 \times 10^{10} \mathrm{M}^{-1} \mathrm{~cm}^{-1}$ at $404 \mathrm{~nm}$.

Silver-coated magnetic nanoparticles (Ag@MNP) were prepared using a co-precipitation method for the synthesis of the

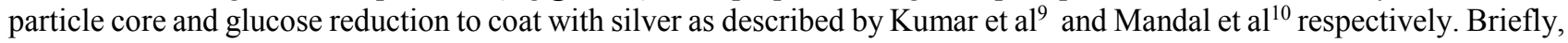
a stock solution of maghemite $\left(\gamma-\mathrm{Fe}_{2} \mathrm{O}_{3}\right)$ nanoparticles was prepared by adding $25 \mathrm{~mL}$ of an acidified iron salt solution $\left(0.4 \mathrm{M} \mathrm{Fe}^{2+}, 0.8 \mathrm{M} \mathrm{Fe}^{3+}, 1 \mathrm{M} \mathrm{HCl}\right)$ drop-wise to $250 \mathrm{~mL}$ of $1.5 \mathrm{M} \mathrm{NaOH}$ at $50^{\circ} \mathrm{C}$ under vigorous non-magnetic stirring. After 20 minutes the particles were allowed to cool, washed twice with DI water and once with $0.1 \mathrm{M} \mathrm{HNO}_{3}$. An additional $125 \mathrm{~mL}$ of $\mathrm{HNO}_{3}$ is then added to the solution, stirred an additional 40 minutes at $95^{\circ} \mathrm{C}$, and resuspended in distilled water. 
To coat with silver, $1 \mathrm{~mL}$ of these stock MNPs was mixed with $4 \mathrm{~mL}$ of $0.35 \mathrm{M}$ glucose and $1.5 \mathrm{~mL}$ of $60 \mu \mathrm{M} \mathrm{AgNO}$. The solution was sonicated for 10 minutes then heated to $90^{\circ} \mathrm{C}$ for 90 minutes. Finally, the particles were centrifuged three times to wash, and redispersed in $6 \mathrm{~mL}$ of $5 \mathrm{mM}$ sodium citrate for a particle diameter of $\sim 31 \mathrm{~nm}$ and final concentration of $608 \mathrm{pM}$ as previously reported ${ }^{11}$.

\subsection{Target and Probe Nanoparticle Conjugation}

\section{Aptamer/RRM AgNP Target Synthesis}

Prior to immobilization, the BPA aptamers were suspended in a $60 \mathrm{mM}$ phosphate buffer (PBS, $\mathrm{pH} 8.5$ ) and treated with $15 \mathrm{mg}$ of dithiothreitol (DTT) to reduce their disulfide bonds. After one hour the aptamers were HPLC purified to remove residual DTT, and then heated to $90^{\circ} \mathrm{C}$ for 5 minutes to fold the aptamers into their tertiary structure for optimal binding.

Target AgNPs were functionalized with the aptamer in accordance with the method developed by Zhang et al ${ }^{12}$. The aptamer $(7 \mu \mathrm{M})$ was added to $1 \mathrm{~mL}$ of silver colloid $(145 \mathrm{pM})$ at a molar ratio of 2000:1 and left for 1 hour. Three $20 \mu \mathrm{L}$ aliquots of $250 \mathrm{mM}$ citrate $\mathrm{HCl}$ buffer ( $\mathrm{pH} 2.9$ ) were then added 5 minutes apart, and the sample was left an additional 30 minutes before centrifuging and resuspending the aptamer functionalized particles in $1 \mathrm{~mL}$ of $0.3 \mathrm{M} \mathrm{PBS}(\mathrm{pH}$ 7.4). The Raman reporter molecule, malachite green isothiocyanate (MGICT, $10 \mu \mathrm{M}$ in methanol), was then added at a 500:1 ratio and left to react with the aptamer AgNPs under sonication for 1 hour, which were then centrifuged and stored in a $0.1 \mathrm{M}$ PBS buffer (pH 7.4).

\section{AnalyteAg@MNP Probe Synthesis}

To synthesize $1 \mathrm{~mL}$ of BADGE functionalized magnetic nanoprobes, the $\mathrm{SH}-\mathrm{PEG}-\mathrm{NH}_{2}$ linker was added drop-wise to an excess of the analyte BADGE in 0.3 M PBS buffer ( $\mathrm{pH} 8.5$ ) and left overnight to allow conjugation between their terminal amine and epoxide groups respectfully. The conjugates were then treated with aminoethanol to open unbound epoxide rings and prevent non-specific binding of BADGE to the RRM's amine groups. These SH-PEG-BADGE conjugates were then added at a 5000:1 ratio to $1 \mathrm{~mL}$ of silver coated magnetic nanoparticles (diluted to $200 \mathrm{pM}$ ). After one hour three $20 \mu \mathrm{L}$ aliquots of $250 \mathrm{mM}$ citrate $\mathrm{HCl}$ buffer were again added 5 minutes apart, and the sample was left an additional 30 minutes before centrifuging and resuspending the BADGE functionalized particles in $1 \mathrm{~mL}$ of $0.1 \mathrm{M}$ PBS (pH 7.4).

\subsection{SERS analysis}

Equal volumes containing $30 \mathrm{pM}$ of each of the target and probe nanoparticles were left overnight to allow complete binding between the immobilized BPA aptamers and BADGE. The assembled nanoparticle clusters were collected using a neodymium magnet held at the side of the glass vial for 30 minutes, the supernatant containing any unbound AgNPs was removed, and the assembled nanoparticles were resuspended in 0.1 M PBS (pH 7.4). All SERS measurements were collected with the target AgNP concentration held constant at $7.5 \mathrm{pM}$ in a total volume of $30 \mu \mathrm{L}$ of $0.1 \mathrm{M}$ PBS (pH 7.4) with an integration time of 10 seconds ( $1 \mathrm{~s}$ exposure). For competitive binding analysis $15 \mu \mathrm{L}$ of the assembled target/probe NPs was mixed with $15 \mu \mathrm{L}$ of $1 \mu \mathrm{M}$ BPA in $0.1 \mathrm{M}$ PBS (pH 7.4) and SERS measurements were taken every 30 seconds.

\section{RESULTS AND DISSCUSSION}

The target and probe nanoparticles were functionalized as described and yielded average zeta potential measurements of 39.7 and $-34.6 \mathrm{mV}$ respectively, indicating that the PEG spacer and the HEG modified aptamer provide adequate particle stabilization in the $0.1 \mathrm{M}$ PBS binding buffer. The intrinsic SERS signal of a stock solution of the the assembled nanoparticles and its response to the presence of competing BPA was then analyzed. As shown in Figure 2, the SERS signal of the reporter molecule MGICT increases by two orders of magnitude as it experiences an enhancement in the electric field intensity of its immediate environment due to the aptamer-binding induced particle aggregation. When free BPA is added to the conjugate solution, it is observed that the SERS intensity of MGICT's $1175 \mathrm{~cm}^{-1}$ peak (aromatic C$\mathrm{H}$ bending vibrational mode ${ }^{13}$ ) decreases to a steady state over the course of $\sim 5 \mathrm{~min}$ as competitive binding occurs (Fig. 2, inset). That is to say, the BPA aptamer immobilized on the target AgNPs releases from the BADGE on the MNPs in order to more favorably bind to free BPA, and the released particles return to colloidal suspension causing a decrease in the solution SERS signal as hypothesized. 


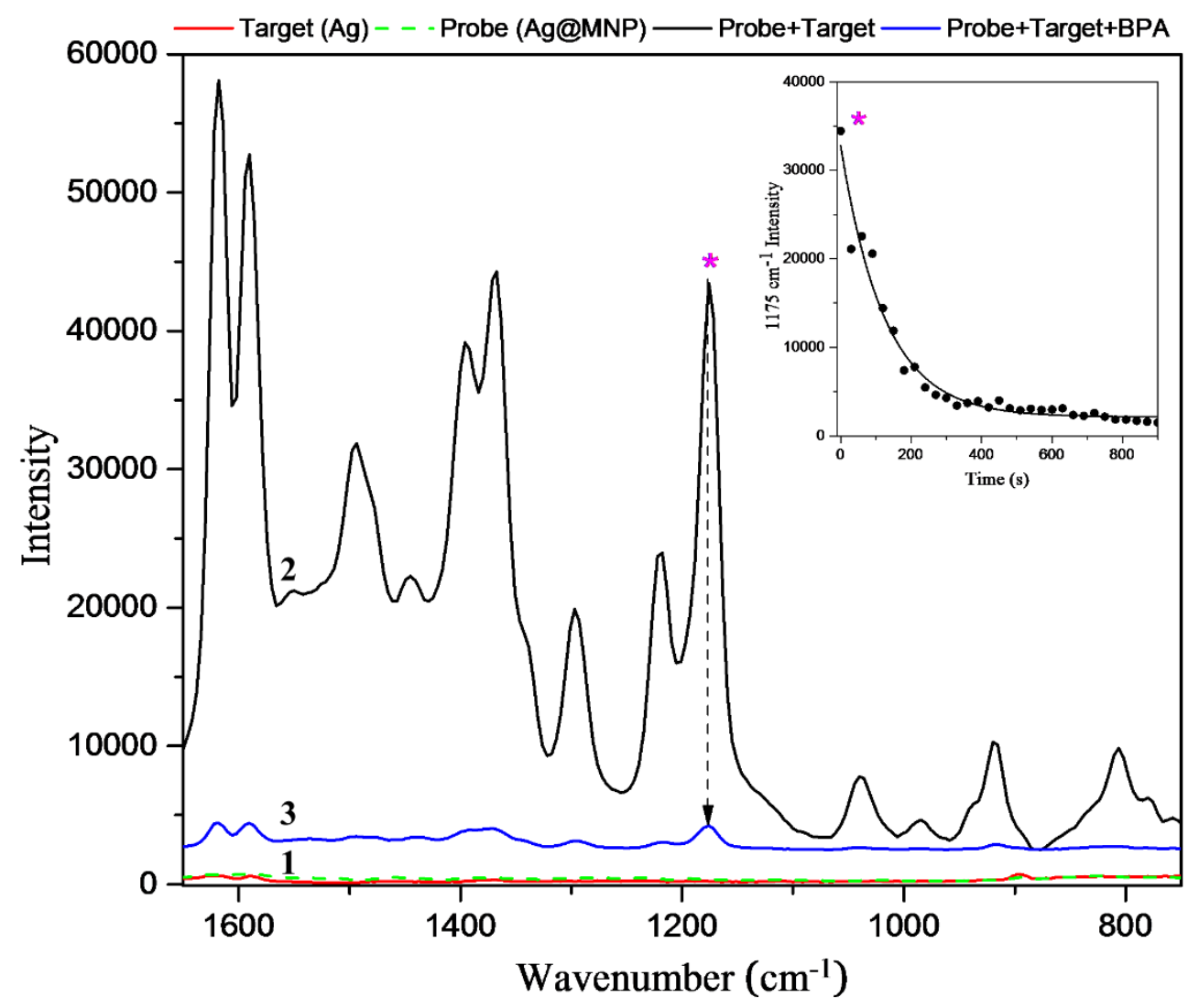

Figure 2. SERS spectra of the target and probe nanoparticles: (1) individually, (2) mixed overnight to allow complete NP assembly via aptamer/BADGE binding, and (3) assembled NPs after exposure to $15 \mathrm{pmol}$ of the competing analyte. Inset: SERS monitoring of the competitive binding event over $15 \mathrm{~min}$.

\section{CONCLUSION}

In summary, we have described a "turn-off" SERS assay platform methodology for the detection of small toxins utilizing aptamer-mediated assembly of SERS active colloidal nanoparticles. The SERS assay was able to monitor a five minute competitive binding event initiated by the presence of 15 pmols bisphenol A in free solution. However, detailed characterization of the dose-response relationship and coefficients of variation of the system will assist in further development of this molecular recognition platform. Additionally, this approach has the potential to be translated to any aptamer/antigen pair and provides the added benefit of magnetic manipulation of the nanoparticle sensing network.

\section{REFERENCES}

1. Moghaddam, A.; Løbersli, I.; Gebhardt, K.; Braunagel, M.; Marvik, O. J., Selection and characterisation of recombinant single-chain antibodies to the hapten Aflatoxin-B1 from naive recombinant antibody libraries. Journal of Immunological Methods 2001, 254 (1-2), 169-181.

2. Jayasena, S. D., Aptamers: An emerging class of molecules that rival antibodies in diagnostics. Clinical Chemistry 1999, 45 (9), 1628-1650.

3. (a) Graham, D.; Stevenson, R.; Thompson, D. G.; Barrett, L.; Dalton, C.; Faulds, K., Combining functionalised nanoparticles and SERS for the detection of DNA relating to disease. Faraday discussions 2011, 149, 291-9; discussion 333-56; (b) Guerrini, L.; Graham, D., Molecularly-mediated assemblies of plasmonic nanoparticles for Surface-Enhanced Raman Spectroscopy applications. Chemical Society Reviews 2012, 41 (21), 7085-7107. 
4. Gracie, K.; Correa, E.; Mabbott, S.; Dougan, J. A.; Graham, D.; Goodacre, R.; Faulds, K., Simultaneous detection and quantification of three bacterial meningitis pathogens by SERS. Chemical Science 2014, 5 (3), $1030-1040$.

5. (a) Qu, G.; Zhang, G.; Wu, Z.; Shen, A.; Wang, J.; Hu, J., A "turn-off" SERS assay of heparin with high selectivity based on heparin-peptide complex and Raman labelled gold nanoparticles. Biosens Bioelectron 2014, 60, 124-9; (b) Wu, Z.; Liu, Y.; Zhou, X.; Shen, A.; Hu, J., A "turn-off" SERS-based detection platform for ultrasensitive detection of thrombin based on enzymatic assays. Biosens Bioelectron 2013, 44, 10-5.

6. Marks, H. L.; Pishko, M. V.; Jackson, G. W.; Coté, G. L., Rational Design of a Bisphenol A Aptamer Selective Surface-Enhanced Raman Scattering Nanoprobe. Anal Chem 2014, 86 (23), 11614-11619.

7. $\quad$ Jo, M.; Ahn, J. Y.; Lee, J.; Lee, S.; Hong, S. W.; Yoo, J. W.; Kang, J.; Dua, P.; Lee, D. K.; Hong, S.; Kim, S., Development of single-stranded DNA aptamers for specific bisphenol a detection. Oligonucleotides 2011, 21 (2), 85-91.

8. Leopold, N.; Lendl, B., A new method for fast preparation of highly surface-enhanced Raman scattering (SERS) active silver colloids at room temperature by reduction of silver nitrate with hydroxylamine hydrochloride. The Journal of Physical Chemistry B 2003, 107 (24), 5723-5727.

9. Kumar, G.; Rangarajan, N.; Sonia, B.; Deepika, P.; Rohman, N.; Narayana, C., Metal-coated magnetic nanoparticles for surface enhanced Raman scattering studies. Bull Mater Sci 2011, 34 (2), 207-216.

10. Mandal, M.; Kundu, S.; Ghosh, S. K.; Panigrahi, S.; Sau, T. K.; Yusuf, S. M.; Pal, T., Magnetite nanoparticles with tunable gold or silver shell. J Colloid Interf Sci 2005, 286 (1), 187-194.

11. Donnelly, T.; Smith, W. E.; Faulds, K.; Graham, D., Silver and magnetic nanoparticles for sensitive DNA detection by SERS. Chem Commun 2014.

12. Zhang, X.; Servos, M. R.; Liu, J., Fast pH-assisted functionalization of silver nanoparticles with monothiolated DNA. Chem. Commun. 2012, 48 (81), 10114-10116.

13. Power, A. C.; Betts, A. J.; Cassidy, J. F., Non aggregated colloidal silver nanoparticles for surface enhanced resonance Raman spectroscopy. Analyst 2011, 136 (13), 2794-2801. 